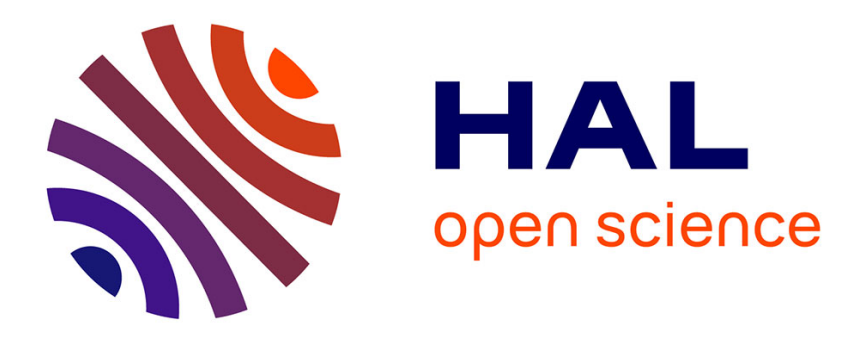

\title{
Mémorisation et traitement statistique d'impulsions laser déclenchées
}

\author{
J.R. Lalanne, J.J. Piaud
}

\section{To cite this version:}

J.R. Lalanne, J.J. Piaud. Mémorisation et traitement statistique d'impulsions laser déclenchées. Revue de Physique Appliquée, 1971, 6 (2), pp.143-145. 10.1051/rphysap:0197100602014300 . jpa00243514

\section{HAL Id: jpa-00243514 https://hal.science/jpa-00243514}

Submitted on 1 Jan 1971

HAL is a multi-disciplinary open access archive for the deposit and dissemination of scientific research documents, whether they are published or not. The documents may come from teaching and research institutions in France or abroad, or from public or private research centers.
L'archive ouverte pluridisciplinaire HAL, est destinée au dépôt et à la diffusion de documents scientifiques de niveau recherche, publiés ou non, émanant des établissements d'enseignement et de recherche français ou étrangers, des laboratoires publics ou privés. 


\title{
MÉMORISATION ET TRAITEMENT STATISTIQUE D'IMPULSIONS LASER DÉCLENCHÉES
}

\author{
par J. R. LALANNE et J. J. PIAUD
}

Centre de Recherches Paul Pascal, C. N. R. S., 33, Talence

(Reçu le 30 septembre 1970, révisé le 7 décembre 1970)

\begin{abstract}
Résumé. - Nous avons mis au point un dispositif entièrement automatisé permettant la mémorisation, l'affichage numérique et l'acquisition sur ordinateur d'impulsions laser déclenchées. Le pilotage de cette expérience (diffusion spontanée linéaire ou non linéaire) est directement assuré par l'ordinateur.
\end{abstract}

Abstract. - Linear and non linear scattered laser waves can be detected and held by an apparatus that we have builded. The signals are sent to a computer which directly fires the Q-switched laser and performs calculations with the stored entries.

1. But de la réalisation. - La diffusion spontanée des ondes laser harmoniques par les liquides permet l'étude des polarisabilités optiques représentées par des tenseurs d'ordre supérieur à deux [1], [2], [3]. Malheureusement, elle se heurte à une difficulté majeure : les fluctuations d'amplitude du signal diffusé dues à sa petitesse d'une part (domaine de la dizaine de photo-électrons) et aux fluctuations intermodes de l'onde incidente [4], d'autre part. La courbe tracée sur la figure 1 (56 impulsions laser) illustre une telle

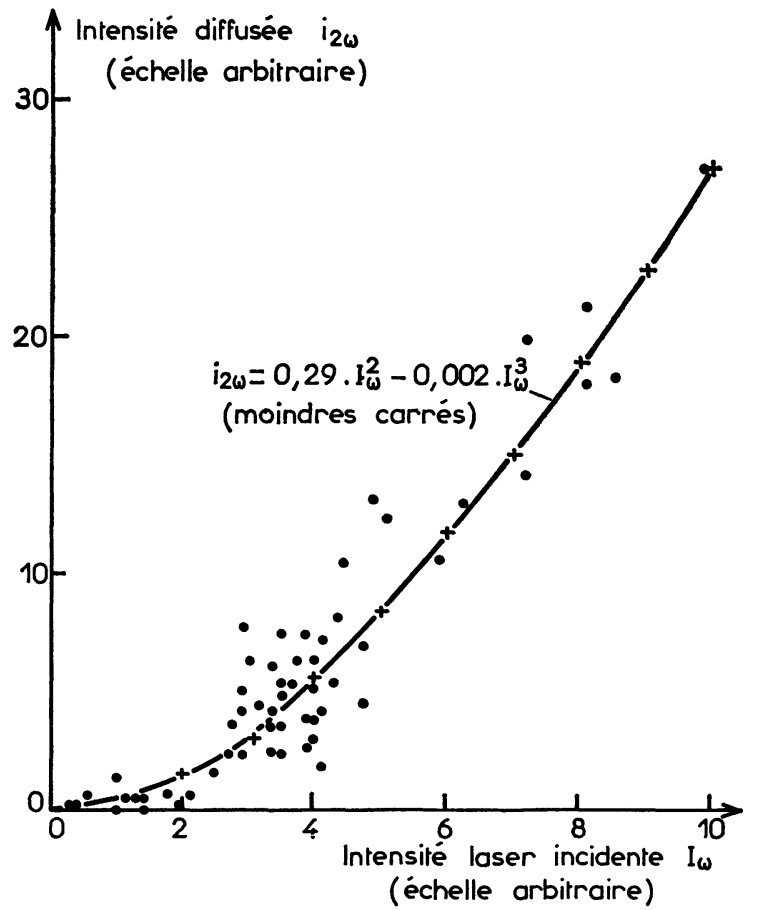

Fig. 1. - Diffusion harmonique (3 $471 \AA$ ) du tétrachlorure de carbone liquide soumis à l'onde laser à rubis déclenché (6 942 A). La détection est faite par oscilloscope, enregistrement photographique et mesure de l'amplitude des impulsions après agrandissement. L'ajustement, par la méthode des moindres carrés, permet une bonne vérification de la dépendance $i \alpha I^{2}$ (le terme $-0,002 I^{3}$ conduit à une correction relative toujours inférieure à $6 \%$. tendance. Il convient, par conséquent, de multiplier les mesures et de les traiter statistiquement afin de pouvoir en déduire une information valable. Cette multiplication impose une expérimentation longue et particulièrement pénible (temps mort de deux minutes entre chaque impulsion laser afin d'assurer le refroidissement; enregistrement photographique ; développement et agrandissement ; mesure des amplitudes; introduction des résultats sur calculatrice). Nous avons cherché à l'automatiser intégralement.

2. Montage expérimental. - La figure 2 présente notre réalisation.

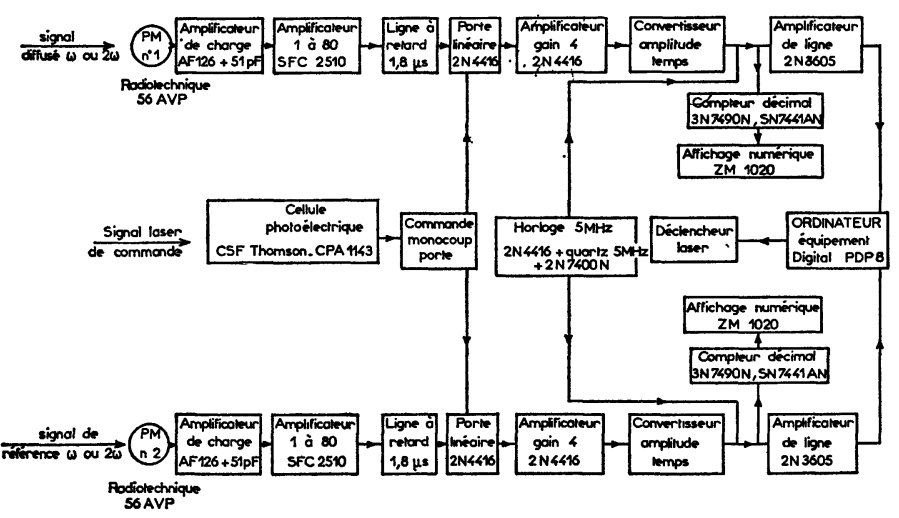

Fig. 2. - Schéma d'ensemble de notre réalisation.

2.1 DéClenChEMENT DU LASER. - Toutes les deux minutes l'ordinateur Equipement Digital PDP8, situé à environ vingt-cinq mètres de notre montage, délivre une impulsion qui, après transformation, déclenche l'impulsion T. H. T. appliquée au réflecteur de notre laser (10 MW, déclenchement par absorbeur saturable, durée à mi-hauteur de l'impulsion : $30 \mathrm{~ns}$ ).

2.2 MéMORISATION DES SIGNAUX. - Le signal diffusé est détecté par le photomultiplicateur $n^{\circ} 1$, convenablement filtré. Corrélativement, un signal de référence linéaire au quadratique, est délivré par le photomulti- 


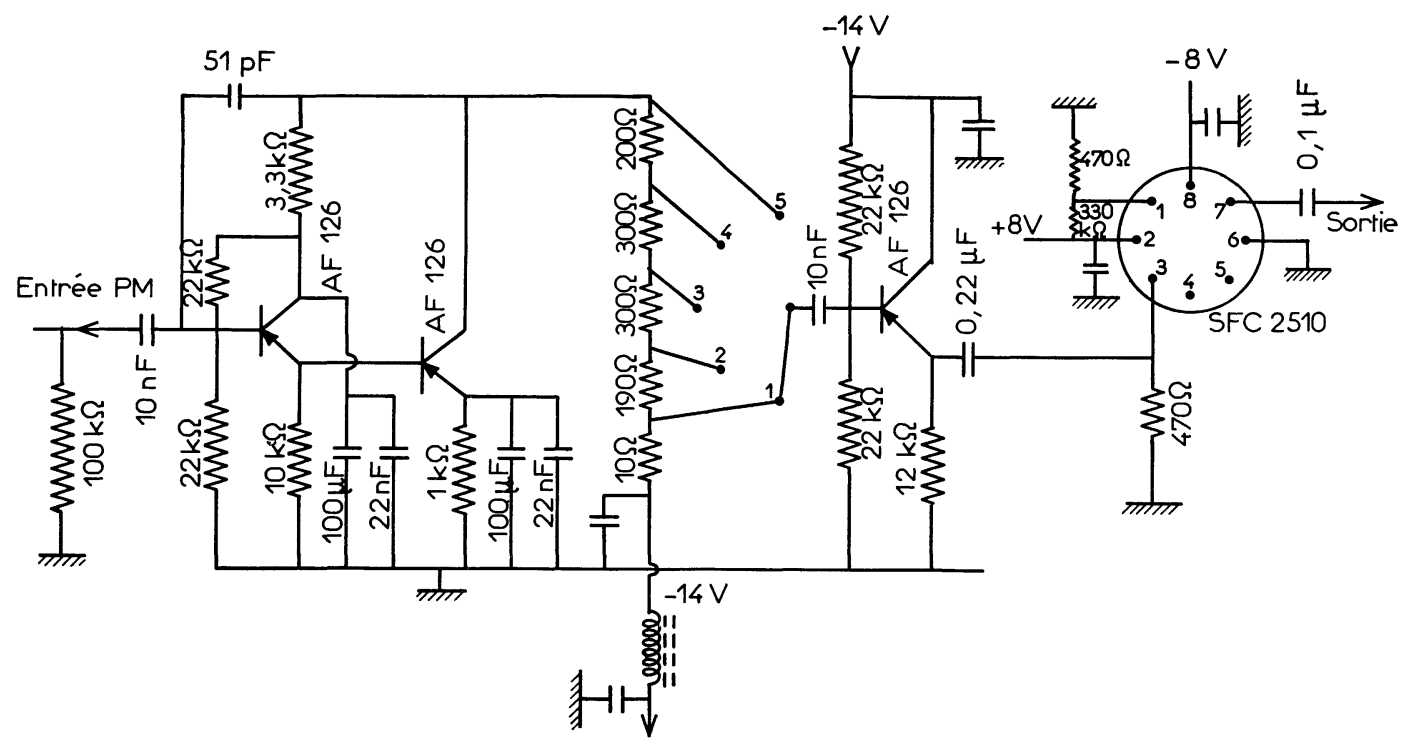

FIG. 3. - Amplificateur de charge et amplificateur de gain réglable entre 0.8 et 80 (5 positions : 0-16-40-64-80).

plicateur $\mathrm{n}^{0}$ 2. L'impédance de charge des deux photomultiplicateurs est de $100 \mathrm{k} \Omega$. Les impulsions électriques attaquent deux amplificateurs de charge (Fig. 3). Ces amplificateurs délivrent des signaux d'amplitudes proportionnelles aux charges électriques transportées par les impulsions des photomultiplicateurs. Les oscillogrammes enregistrés confirment une décroissance temporelle exponentielle de constante de temps peu différente de $10 \mu \mathrm{s}$. Ces signaux sont ensuite amplifiés (gain réglable entre 0.8 et 80 ) (Fig. 3) retardés (ligne à retard de $1,8 \mu \mathrm{s}$ ) puis enfin à nouveau amplifiés (gain 4).

2.3 SéleCtion Des SIGNAUX UtiLes. - Le signal détecté se situant au niveau du bruit, il importe d'utiliser une méthode de coïncidence qui permettra d'effectuer un triage de l'impulsion utile. Pour ce faire, une cellule photo-électrique rapide éclairée par une partie de l'onde laser incidente, pilote une commande. Celle-ci ouvre une porte linéaire dont le temps d'ouverture est inférieur au retard volontairement introduit sur les signaux. La commande a été rendue monocoup afin d'éviter la détection éventuelle de plusieurs impulsions laser autorisées par le déclencheur saturable lorsque le niveau de pompage du laser est trop élevé.

2.4 AfFichage DE L'INFormation AU NIVEAU DU DISPOSITIF EXPÉRIMENTAL. - Nous avons choisi une conversion amplitude-temps effectuée par décharge d'un condensateur à courant constant. Le temps de décharge du condensateur, proportionnel à l'amplitude du signal fourni par les amplificateurs de charge, est mesuré à l'aide d'un comparateur différentiel rapide débloquant une horloge à quartz $(5 \mathrm{MHz})$. Les impulsions sont comptées et le résultat est affiché en décimal au niveau de notre dispositif. Une telle procédure permet une mise au point aisée de l'expérience et un contrôle du système d'acquisition en cours d'expérimentation.
2.5 ACQUisition ET TRAITEMENT STATISTIQUe DES RÉSULTATS EXPÉRIMENTAUX. - Corrélativement à leur affichage numérique, les trains d'impulsions fournis par les horloges sont, après amplification, adressés à

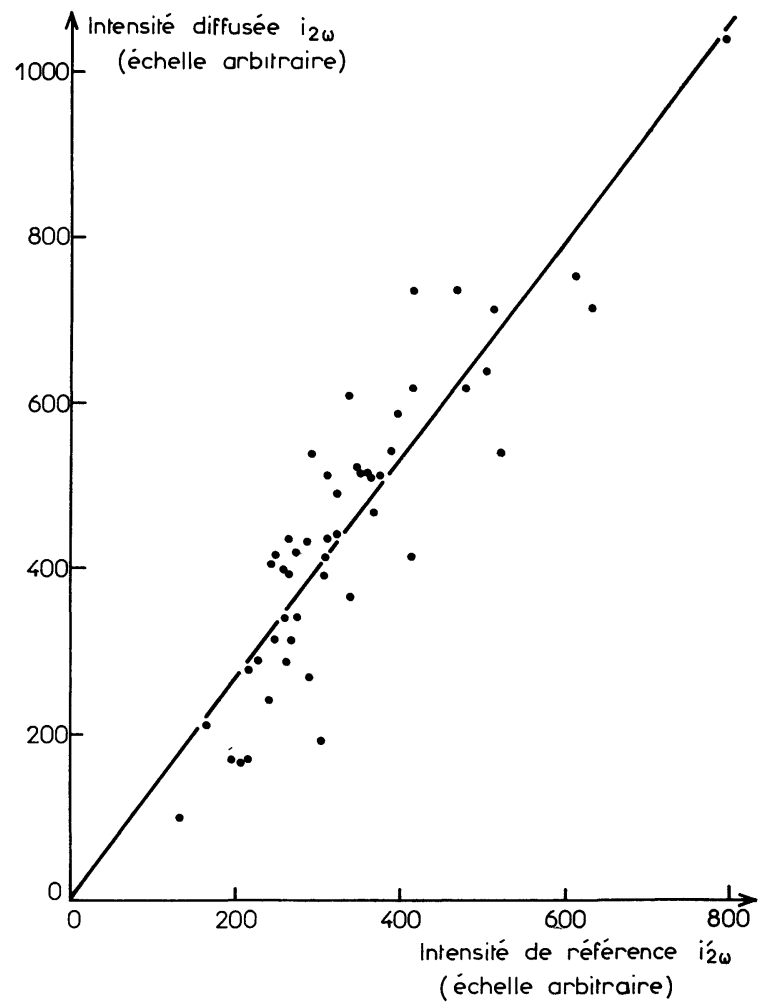

FIG. 4. - Diffusion harmonique Vh $(3471 \AA)$ du tétrachlorure de carbone liquide soumis à l'onde laser à rubis déclenché $(6942 \AA)$. La détection est faite, par rapport à une référence quadratique, par notre montage automatisé. L'ajustement linéaire par la méthode des moindres carrés conduit aux valeurs : Coefficient de corrélation : $r^{2}=0,66$; Coefficient de régression : $b=1,33$; Ecart-type relatif estimé sur une population infinie

$$
\frac{S_{b^{2}}}{b^{2}}=10^{-1} \text {. }
$$


un ordinateur Equipement Digital PDP8 qui assure leur comptage et permet leur traitement ultérieur.

3. Caractéristiques techniques de notre réalisation. - 3.1 SENSIBILITÉ DES AMPLIFICATEURS DE CHARGE. Nous avons mesuré, dans les conditions d'expérimentation, les taux de conversion au niveau des amplificateurs de charge.

Amplificateur de charge 1 :

$$
(0,75 \pm 0,20) 10^{10} \mathrm{~V} \times \mathrm{C}^{-1}
$$

Amplificateur de charge 2 :

$$
(0,80 \pm 0,20) 10^{10} \mathrm{~V} \times \mathrm{C}^{-1}
$$

3.2 DYNAMIQUe ET LINÉARITÉ. - La puissance laser varie considérablement d'une impulsion à une autre (au maximum d'un facteur 3). Nous avons, par conséquent, imposé à notre montage une dynamique de 10 . Nos vérifications garantissent une linéarité suffisante $(>90 \%)$ sur toute l'échelle de mesure.

3.3 Limites DE DÉTECTION DE NOTRE MONTAGE. A la sortie de l'amplificateur à gain réglable, notre systèmedétec te les impulsions d'amplitude comprises entre 0,25 et $2.5 \mathrm{~V}$. Compte tenu du gain compris entre 0.8 et 80 , les limites inférieure et supérieure de détection deviennent respectivement $3 \mathrm{mV}$ et $3 \mathrm{~V}$. Avec un taux de conversion moyen de

$$
0,75 \times 10^{10} \mathrm{~V} \times \mathrm{C}^{-1}
$$

ces limites correspondent à $4 \times 10^{-13} \mathrm{C}$ (soit $2,5 \times 10^{6}$ électrons) et $4 \times 10^{-10} \mathrm{C}$ (soit $2,5 \times 10^{9}$ électrons). Ces nombres montrent qu'avec des photomultiplicateurs de gain moyen $10^{6}$, on pourra détecter des impulsions comprises entre quelques photo-électrons et 2500 photo-électrons.

3.4 EXEMPle DE DÉTECTION. - Afin de tester notre montage, nous avons répété l'expérience de diffusion dont les résultats ont été reportés sur la figure 1. Les nouveaux résultats (50 impulsions laser - durée de l'expérience : $2 \mathrm{~h}$ ) sont reportés sur la figure 4. La référence choisie, cette fois quadratique, est obtenue par diffusion harmonique d'une lame de K. D. P. placée sur le trajet de l'onde rouge. Les deux signaux de diffusion harmonique doivent, bien entendu, être proportionnels.

Les résultats obtenus traduisent cette proportionnalité. La dispersion des points est très satisfaisante, compte tenu de la faiblesse des signaux enregistrés, et n'excède pas celle obtenue par mesure directe des amplitudes sur l'oscillogramme.

Cet appareillage permet d'entreprendre l'étude approfondie de la diffusion non linéaire des molécules.

Remerciements. - Nous tenons à remercier MM. Fug et Sanchez, Ingénieurs au Centre National de la Recherche Scientifique, qui ont assuré avec nous le couplage de notre manipulation à l'ordinateur.

\section{Bibliographie}

[1] Terhune (R. W.), Maker (P. D.) et Savage (C. M.), Phys. Rev. Letters, 1965, 14, 681.

[2] Maker (P. D.), Phys. Rev., 1970, A, 1, 923.
[3] Kielich (S.), Lalanne (J. R.) et Martin (F. B.), à paraître dans Phys. Rev. Letters, 1971.

[4] Ducuing (J.) et Bloembergen (N.), Phys. Rev., 1964, 133, 6 A, 1493. 